\title{
Surface fitting with boundary data
}

\author{
Sara Maad*, Clyde Martin ${ }^{\dagger}$, Thomas Strömberg ${ }^{\ddagger}$, Johan Byström ${ }^{\S}$ \\ * Department of Mathematics \\ P. O. Box 400137 (Kerchof Hall) \\ University of Virginia \\ Charlottesville, VA 22904-4137 \\ e-mail: sm4tc@virginia.edu \\ $\dagger$ Department of Mathematics \\ Texas Tech University \\ Lubbock, Texas 79409 \\ e-mail: martin@math.ttu.edu \\ $\ddagger$ Luleå tekniska universitet \\ Institutionen för matematik \\ Universitetsområdet, Porsön \\ SE-971 87 Luleå \\ Sweden \\ e-mail: strom@sm.luth.se \\ $\S$ Luleå tekniska universitet \\ Institutionen för matematik \\ Universitetsområdet, Porsön \\ SE-971 87 Luleå \\ Sweden \\ e-mail: johanb@sm.luth.se
}

Keywords. Surface fitting, data, splines

\begin{abstract}
The problem of fitting surfaces to data is a well studied problem in statistics. However when there is prior information the theory is not developed. It often happens in toxicology and in medicine that the effect of a single drug is well understood. However if a pair of drugs is delivered in tandem or if two toxins are interacting the effect is not understood. In this paper we attack the problem of fitting a surface to a data set contained in a square when two of the boundaries are known. Our approach generalizes the concept of smoothing splines.
\end{abstract}

\section{INTRODUCTION}

The theory of approximation of functions of one variable is one of the most studied areas in mathematics and statistics. To this end the theory of polynomial splines and, more recently, the theory of control theoretic splines, [8], [5], [9], [2], has been extensively developed and used in applications. In statistics the method of smoothing splines has been developed based on the work of Grace Wahba [6], and her collaborators. Common to all splines is a data set of the form

$$
D=\left\{\left(t_{i}, \alpha_{i}\right): i=1, \cdots, N\right\}
$$

and a scheme to construct a function $y(t)$ such that either $y\left(t_{i}\right)=\alpha_{i}$ or $y\left(t_{i}\right)-\alpha_{i}$ is small in some precise sense.

Control theoretic splines were developed originally as a tool for control theory to be used in such problems as path planning in robotics and terminal area control of aircraft, [8], [1]. The idea was to use a controlled differential equation to push a curve through a set of desired points. This is not far from the original idea of splines and is very close to the development of smoothing splines developed by Wahba. Here the idea is to use a system of the form

$$
\dot{x}=A x+b u, \quad y=c x
$$

and then to choose a cost function that controls the input function $u$. The original development of splines used the following:

$$
\ddot{x}(t)=u(t), \quad y=x
$$

with $y\left(t_{i}\right)=\alpha_{i}$ and $\sup _{t}|u(t)|$ is minimized. Smoothing splines used a quite different concept of "small". In Wahba's original formulation the idea was to solve the following optimization problem:

$$
\min _{u}\left[\int_{0}^{T} u^{2}(t) d t+\sum_{i=1}^{N}\left(y\left(t_{i}\right)-\alpha_{i}\right)^{2}\right]
$$

where

$$
\ddot{x}=u, \quad y=x .
$$

These two problems are quite different but have almost the same solution. In fact both problems are solved in a suitable Hilbert space. 
In this paper we use the techniques of optimal splines to develop a method of fitting surfaces to data. We do not consider the classical problem of fitting a surface to data points but consider a problem that arises in testing drugs and also arises in toxicology. We often know the effect of a single drug or toxin on some biomarker, but little may be known about the effect of combining two drugs or what the effect of the two drugs will be on the same biomarker. It is often that we have need to know what mixture of the two drugs will be the most effective. Thus we are presented with a problem of surface fitting in which we know a great deal about the boundary of the surface but know little about the interior. The cost of doing multiple experiments with combinations of drugs is expensive in time and laboratory costs. Thus there may be only a few points known in the interior region. We will use a 2-dimensional generalization of smoothing splines to construct such surfaces.

After completion of this manuscript, it came to our attention that Wahba has some papers about surface fitting which are relevant for us, and actually quite close to what we study. We would like to thank referee \#3 for pointing this out. In the paper [7], Wahba studies a more general problem, including a similar minimization problem as we study. The difference is that while Wahba minimizes the functional over the whole Sobolev space $H^{2}(S)$ (see section III for a definition), we perform the optimization in an affine subspace of $H^{2}(S)$, which is the space of functions in $H^{2}(S)$ having specified boundary values along two of the sides of the square.

In Section 2 we recall Wahba's smoothing splines, and see that these can be obtained from a variational approach. In Section 3 we formulate the surface fitting problem as a problem in the control of partial differential equations and show that the problem reduces to finding the Green's function for the bilaplacian with nonstandard boundary conditions. In Section 4 we compute a numerical solution in a specific example.

\section{SPLINES IN ONE DIMENSION}

We start with a recollection of Wahba's smoothing splines. The given data is $\left\{x_{i}, \alpha_{i}\right\}_{i=1}^{N}$. Let $\lambda>0$ be a fixed constant. The problem is to minimize the entity

$$
\frac{\lambda}{2} \int_{0}^{1} u(x)^{2} d x+\frac{1}{2} \sum_{i=1}^{N}\left(w\left(x_{i}\right)-\alpha_{i}\right)^{2}
$$

among the solutions of

$$
w^{(2)}=u,
$$

where $u \in L^{2}(0,1)$ is an unknown function. In our two dimensional problem we will have prescribed boundary values on some part of the boundary, and have unprescribed values at some other part. To prepare for this situation, we assume that the boundary at $x=0$ is fixed. For simplicity we choose $w(0)=0$. At $x=1$ we leave $w$ unspecified.
Since $u \in L^{2}(0,1)$, it will be convenient to work in the Sobolev space $H^{2}(0,1)=W^{2,2}(0,1)$, which is the closure of $C^{\infty}(0,1)$ in the norm

$\|w\|_{H^{2}}=\left(\int_{0}^{1}\left(w(x)^{2}+w^{(1)}(x)^{2}+w^{(2)}(x)^{2}\right) d x\right)^{1 / 2}$.

We will refer to $H^{2}(0,1)$ simply as $H$. As is customary, we identify $w \in H^{2}(0,1)$ which are equal almost everywhere. Moreover, our boundary condition requires that we look for solutions $w$ in the space $H$ which is the closure of

$$
\left\{w \in C^{\infty}(0,1) ; w \equiv 0 \text { in some interval }(0, \delta)\right\}
$$

in the $H^{2}$-norm.

By substituting 2 into 1 , we see that the problem is equivalent to that of minimizing

$$
J(w)=\frac{\lambda}{2} \int_{0}^{1} w^{(2)}(x)^{2} d x+\frac{1}{2} \sum_{i=1}^{N}\left(w\left(x_{i}\right)-\alpha_{i}\right)^{2}
$$

among all functions in $H$.

Let $v \in C^{\infty}(0,1)$ be a fixed function such that $v \equiv 0$ on some interval $(0, \delta)$. Then for each $\epsilon>0$, the functions $w+\epsilon v \in H$. Moreover,

$$
\begin{aligned}
\frac{d}{d \epsilon} J(w+ & \epsilon v)\left.\right|_{\epsilon=0}=\lambda \int_{0}^{1} w^{(2)}(x) v^{(2)}(x) d x \\
& +\sum_{i=1}^{N}\left(w\left(x_{i}\right)-\alpha_{i}\right) v\left(x_{i}\right) .
\end{aligned}
$$

If $J(w)$ is minimal, the derivative has to vanish. After two integrations by part we then have

$$
\begin{gathered}
\lambda\left(w^{(2)}(1) v^{(1)}(1)-w^{(3)}(1) v(1)+\int_{0}^{1} w^{(4)}(x) v(x) d x\right) \\
+\sum_{i=1}^{N}\left(w\left(x_{i}\right)-\alpha_{i}\right) v\left(x_{i}\right)=0 .
\end{gathered}
$$

Since $v$ is arbitrary, we see that $w$ has to satisfy the equation

$$
w^{(4)}=0 \quad \text { in }(0,1) \backslash \cup_{i=1}^{N}\left\{x_{i}\right\},
$$

and the boundary conditions

$$
\begin{aligned}
w(0) & =w^{(1)}(0)=0 \\
w^{(2)}(1) & =w^{(3)}(1)=0 .
\end{aligned}
$$

At the points $x_{i}$, we have the conditions

$$
\lambda\left(w^{(3)}\left(x_{i}+\right)-w^{(3)}\left(x_{i}-\right)\right)=-\left(w\left(x_{i}\right)-\alpha_{i}\right) .
$$

Thus, we see that the problem reduces to finding solutions to (3) in each subinterval $\left(0, x_{1}\right),\left(x_{i}, x_{i+1}\right)$ and $\left(x_{N}, 1\right)$. These solutions are third degree polynomials, which at the points $x_{i}$ are fit together so that $w$ is a $C^{2}$ function with jumps in the third derivative given by (4). 


\section{SURFACE FITTING IN TWO DIMENSIONS}

Given two real functions $f$ and $g$, defined on the interval $[0,1]$ and data points $\left\{x_{i}, y_{i}, \alpha_{i}\right\}$, where $\left(x_{i}, y_{i}\right) \in S=$ $(0,1) \times(0,1), i=1 \ldots N$, we would like to find a function $w(x, y) \in C^{2}([0,1] \times[0,1])$ such that

$$
\Delta w=u,
$$

which satisfies the boundary values $w(x, 0)=f(x)$ and $w(0, y)=g(y)$. Moreover, we would like to pick the one solution such that, for a fixed number $\lambda>0$, the entity

$$
\lambda \int_{0}^{1} \int_{0}^{1} u(x, y)^{2} d x d y+\sum_{i=1}^{N}\left(w\left(x_{i}, y_{i}\right)-\alpha_{i}\right)^{2}
$$

is minimal.

The space we work in is the Sobolev space $H^{2}(S)$, and we will denote it with $H$. This space is the closure of $C^{\infty}(S)$ in the norm

$$
\|w\|_{H^{2}}=\left(\sum_{|\alpha| \leq 2} \int_{0}^{1} \int_{0}^{1}\left|D^{\alpha} w\right|^{2} d x d y\right)^{1 / 2} .
$$

As before, we identify $w \in H$ which are equal almost everywhere. The boundary conditions require that we seek solutions $w$ in the affine subspace $M$ of $H^{2}(S)$, which is the closure of

$$
\{w \in C(S) ; w=f(x) \text { in a neighborhood }
$$

of the $x$-axis, and $w=g(y)$

in a neighborhood of the $y$-axis $\}$.

As in Section II, we bypass the control $u$, and minimize the functional

$$
J(w)=\frac{\lambda}{2} \int_{0}^{1} \int_{0}^{1}(\Delta w)^{2} d x d y+\frac{1}{2} \sum_{i=1}^{N}\left(w\left(x_{i}, y_{i}\right)-\alpha_{i}\right)^{2}
$$

among all functions $w \in M$.

Note that $M$ is closed and convex, and so it is weakly closed. The functional $J$ is bounded below, weakly lower semi-continuous, and coercive on $M$. Thus, the direct method of the calculus of variations (see e.g. [4], p. 4) shows that there exists a solution to (5).

Let $v \in C^{\infty}(S)$ be such that $v \equiv 0$ in a neighborhood of the axes $x=0$ and $y=0$. Then the comparison functions $w+\epsilon v$ are also in $M$, and if $J(w)$ is minimal we must have

$$
\left.\frac{d}{d \epsilon} J(w+\epsilon v)\right|_{\epsilon=0}=0,
$$

i.e.

$$
\lambda \int_{0}^{1} \int_{0}^{1} \Delta w \Delta v d x d y+\sum_{i=1}^{N}\left(w\left(x_{i}, y_{i}\right)-\alpha_{i}\right) v_{i}(x)=0 .
$$

After two integrations by part, we then have

$$
\lambda\left(\int_{\partial S} \Delta w \nabla v \cdot \mathbf{n} d s-\int_{\partial S} \nabla(\Delta w) \cdot \mathbf{n} v d s\right.
$$

$\left.+\int_{0}^{1} \int_{0}^{1} \Delta^{2} w v d x d y\right)+\sum_{i=1}^{N}\left(w\left(x_{i}, y_{i}\right)-\alpha_{i}\right) v\left(x_{i}\right)=0$,

where $\mathbf{n}$ is the outward pointing unit normal.

Now, this should hold for all $v$ satisfying the appropriate boundary conditions. Thus, it must in particular hold for all $v \in C^{2}(S)$ which are zero in a neighborhood of $\left(x_{i}, y_{i}\right)$, and which satisfy $v(x, 1)=v(1, y)=0$ and $\nabla v(x, y)=0$ for $(x, y) \in \partial S$. But then $w$ must be biharmonic, i.e.

$$
\Delta^{2} w=0
$$

in $S \backslash \cup_{i=1}^{N}\left\{\left(x_{i}, y_{i}\right)\right\}$. Then, by choosing $v$ appropriately, we obtain the natural boundary conditions

$$
\begin{aligned}
\Delta w(x, 1) & =\Delta w(1, y)=0 \\
\frac{\partial}{\partial x} \Delta w(1, y) & =\frac{\partial}{\partial y} \Delta w(x, 1)=0,
\end{aligned}
$$

together with the imposed boundary condition

$$
\begin{aligned}
w(x, 0) & =f(x), \\
w(0, y) & =g(y), \\
\frac{\partial}{\partial x} w(0, y) & =\frac{\partial}{\partial y} w(x, 0)=0 .
\end{aligned}
$$

Moreover, at the points $\left(x_{i}, y_{i}\right)$, there are singularities in the third derivative:

$$
\lambda\left(\lim _{\epsilon \rightarrow 0} \iint_{B_{\epsilon}\left(x_{i}, y_{i}\right)} \Delta^{2} w d x d y\right)=\alpha_{i}-w\left(x_{i}, y_{i}\right) .
$$

Thus, the problem reduces to that of finding a biharmonic Green's function with mixed boundary conditions. Indeed, let $\left(x_{0}, y_{0}\right) \in S$, and let $G\left(x, y ; x_{0}, y_{0}\right)$ be the biharmonic Green's function with homogeneous boundary conditions of the type as (6) and (7), i.e. let $G\left(x, y ; x_{0}, y_{0}\right)$ be the solution to

$$
\Delta^{2} G\left(x, y ; x_{0}, y_{0}\right)=\delta\left(x-x_{0}, y-y_{0}\right)
$$

for $(x, y) \in S$, and

$$
\begin{aligned}
G\left(0, y ; x_{0}, y_{0}\right) & =G\left(x, 0 ; x_{0}, y_{0}\right)=0, \\
\frac{\partial}{\partial y} G\left(0, y ; x_{0}, y_{0}\right) & =\frac{\partial}{\partial y} G\left(x, 0 ; x_{0}, y_{0}\right)=0 . \\
\Delta G\left(x, 1 ; x_{0}, y_{0}\right) & =\Delta G\left(1, y ; x_{0}, y_{0}\right)=0, \\
\frac{\partial}{\partial y} \Delta G\left(x, 1 ; x_{0}, y_{0}\right) & =\frac{\partial}{\partial x} \Delta G\left(1, y ; x_{0}, y_{0}\right)=0 .
\end{aligned}
$$

Let $H$ be the solution to $\Delta^{2} H=0$ in $S$ with boundary conditions (6) and (7). Then $H$ can be expressed in terms of $G$ via the formula

$$
\begin{gathered}
H(x, y)=\int_{0}^{1} f(x) \frac{\partial}{\partial y} \Delta G(x, 0) d x \\
+\int_{0}^{1} g(y) \frac{\partial}{\partial x} \Delta G(0, y) d y .
\end{gathered}
$$

It is now easy to check that a solution to the original problem must satisfy

$$
w(x, y)=H(x, y)
$$




$$
+\frac{1}{\lambda} \sum_{j=1}^{n}\left(\alpha_{j}-w\left(x_{j}, y_{j}\right)\right) G\left(x, y ; x_{j}, y_{j}\right) .
$$

In particular at $(x, y)=\left(x_{i}, y_{i}\right)$, we have

$$
\begin{gathered}
w\left(x_{i}, y_{i}\right)=H\left(x_{i}, y_{i}\right) \\
+\frac{1}{\lambda} \sum_{j=1}^{n}\left(\alpha_{j}-w\left(x_{j}, y_{j}\right)\right) G_{\left(x_{j}, y_{j}\right)}\left(x_{i}, y_{i}\right),
\end{gathered}
$$

where $H$ is given by (8). This is a finite dimensional linear system, and we can solve for $w\left(x_{i}, y_{i}\right)$ if and only if $-\lambda$ is not an eigenvalue of the symmetric matrix $\left\{G_{x_{i}, y_{i}}\left(x_{j}, y_{j}\right)\right\}$. When this is done, equation (9) together with (8) gives an expression for $w$ in terms of its biharmonic Green's function.

We hope that these calculations show the significance of the biharmonic Green's function in the surface fitting problem. Although it is known that the Green's function exists it is not easy to calculate explicitly for the kind of boundary data that we are interested in. It would be interesting if one could find an integral representation formula for the Green's function on the square, and with the boundary conditions (6) and (7).

\section{Simulations}

Using the MATLAB toolbox FEMLAB we compute a numerical solution in three steps:

1) $G\left(x, y ; x_{i}, y_{i}\right), i=1, \ldots, N$, is computed at the node points of a finite element mesh, which contains the given points $\left(x_{i}, y_{i}\right)$.

2) We solve the biharmonic equation

$$
\Delta^{2} H(x, y)=0,
$$

for $(x, y) \in S$, where $H$ is subject to the same boundary conditions as $w$.

3) Finally we set $w=H+\varphi$, where $\varphi$ solves

$$
\Delta^{2} \varphi(x, y)=\frac{1}{\lambda} \sum_{i=1}^{N}\left(\beta_{i}-\varphi\left(x_{i}, y_{i}\right)\right) \delta\left(x-x_{i}, y-y_{i}\right)
$$

in $S$ for

$$
\beta_{i}=\alpha_{i}-v\left(x_{i}, y_{i}\right) .
$$

Then $\varphi$ is subject to homogeneous boundary conditions and can be written on the form

$$
\varphi(x, y)=\frac{1}{\lambda} \sum_{i=1}^{N}\left(\beta_{i}-\varphi_{i}\right) G\left(x, y ; x_{i}, y_{i}\right)
$$

where the coefficients $\varphi_{j}$ satisfy

$$
\lambda \varphi_{j}=\lambda \varphi\left(x_{j}, y_{j}\right)=\sum_{i=1}^{N}\left(\beta_{i}-\varphi_{i}\right) G\left(x_{j}, y_{j} ; x_{i}, y_{i}\right)
$$

for $j=1 \ldots N$. One can uniquely determine $\varphi_{j}$ from this system of linear equations provided $-\lambda$ is not an eigenvalue of the symmetric matrix whose entries are $G\left(x_{i}, y_{i} ; x_{j}, y_{j}\right)$.
TABLE I

DATA SETS

\begin{tabular}{c||c|c|c||c|c|c||c|c|c||} 
Sample & $x_{1}$ & $y_{1}$ & $\alpha_{1}$ & $x_{2}$ & $y_{2}$ & $\alpha_{2}$ & $x_{3}$ & $y_{3}$ & $\alpha_{3}$ \\
\hline \hline $\mathbf{1}$ & 0.1 & 0.6 & 20 & 0.4 & 0.4 & 30 & 0.8 & 0.7 & 40 \\
\hline $\mathbf{2}$ & 0.1 & 0.6 & 2 & 0.4 & 0.4 & 3 & 0.8 & 0.7 & 4 \\
\hline \hline
\end{tabular}

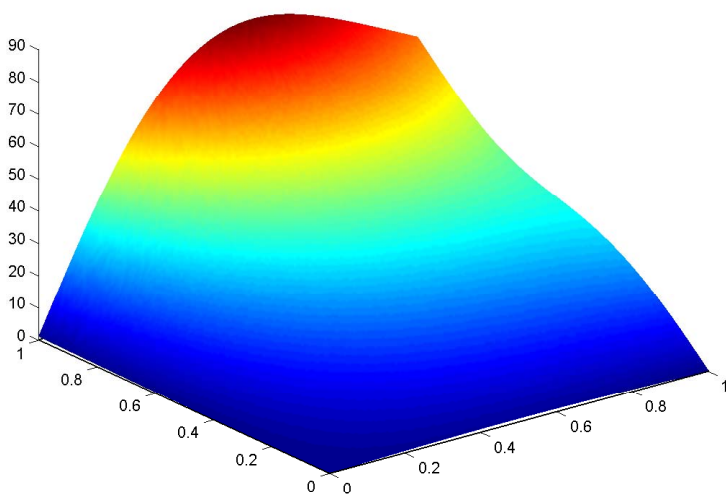

Fig. 1. The graph of $w$ for sample 1

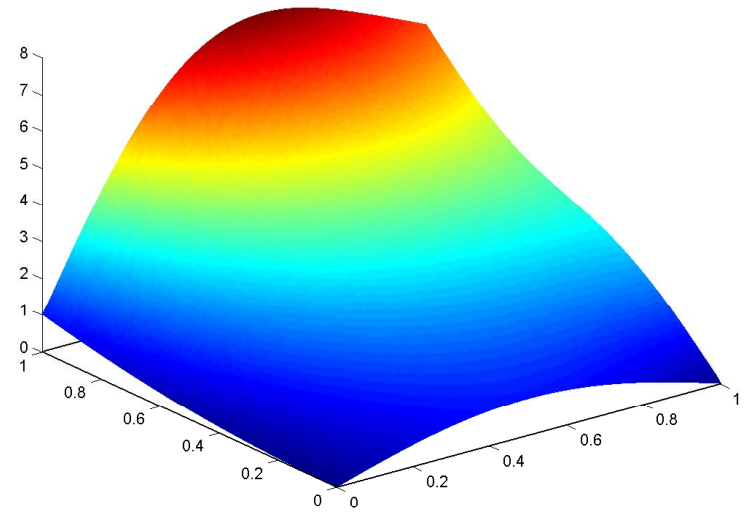

Fig. 2. The graph of $w$ for sample 2

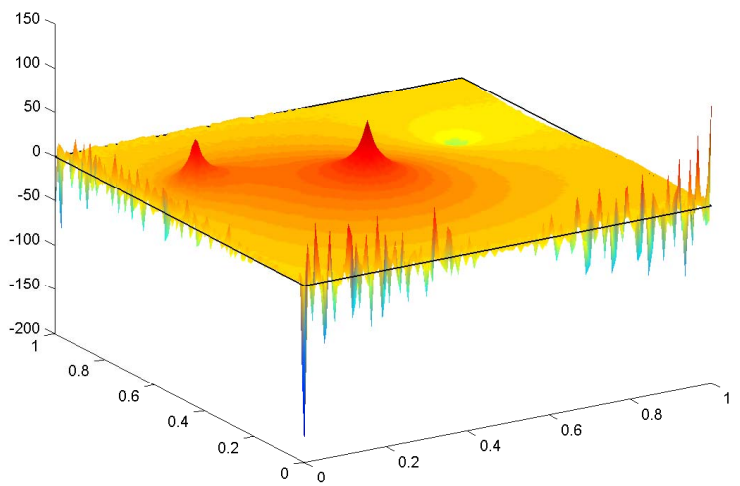

Fig. 3. The graph of the control $u$ for sample 1 


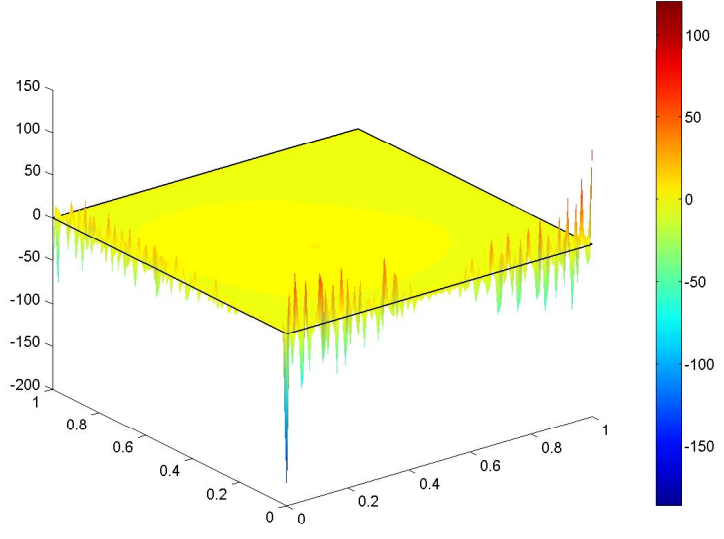

Fig. 4. The graph of the control $u$ for sample 2

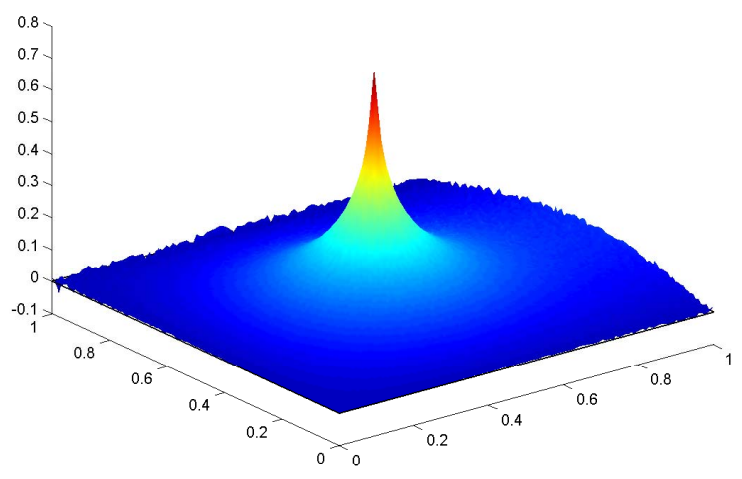

Fig. 5. The laplacian of the Green's function $G\left(x, y ; x_{2}, y_{2}\right)$

We have studied two different data sets, each with three points $\left(x_{i}, y_{i}\right)$. The parameter $\lambda$ is chosen to be 0.1 in both samples, and the boundary conditions are specified by the functions $f(x)=\sin (\pi x)$ and $g(y)=y^{2}$.

Table I shows the data we have used. The graph of $w$ can be seen in Figure 1 and 2, respectively. Although the graphs look rather smooth even at the points $\left(x_{i}, y_{i}\right)$, the

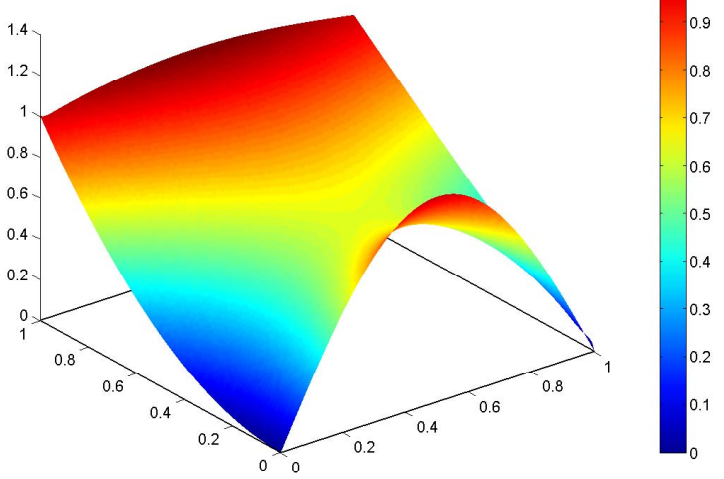

Fig. 6. The biharmonic function $H$

control $u=\Delta w$ has a singularity at $\left(x_{i}, y_{i}\right)$, which is of the order $-\ln r$. This is shown in Figure 3 and 4.

Finally, Figure 5 shows the laplacian of the biharmonic Green's function at point 2, i.e. $\Delta G\left(x, y ; x_{2}, y_{2}\right)$. The biharmonic function $H$ with the specified boundary conditions is shown in Figure 6.

\section{REFERENCES}

[1] Crouch, P.; Jackson, J. Dynamic Interpolation and Application to Flight Control, Journal of Guidance, Control and Dynamics, Vol. 14, pp. 814 - 822,1991.

[2] Egerstedt, M; Martin, C. Optimal trajecory planning and smoothing splines. Automatica 372001 1057-1064

[3] L.C. Evans, Partial differential equations, 1998

[4] M. Struwe, Variational Methods, Applications to nonlinear partial differential equations and Hamiltonian systems, Springer, 1996.

[5] Sun, S.; Egerstedt, M. B.; Martin, C. F. Control theoretic smoothing splines. IEEE Trans. Automat. Control 45 (2000), no. 12, 2271-2279.

[6] Wahba, G. Spline models for observational data. CBMS-NSF Regional Conference Series in Applied Mathematics, 59. Society for Industrial and Applied Mathematics (SIAM), Philadelphia, PA, 1990.

[7] Wahba, G. Surface fitting with scattered noisy data on Euclidean $D$ space and on the sphere, Rocky Mountain Journal of Mathematics, vol. 14, 1984, 281-299.

[8] Z. Zhang, J.Tomlinson, C. Martin, Splines and linear control theory, Acta. Appl. Math., 49, 1997,1-34

[9] Y. Zhou, W. Dayawansa, C. Martin, Control theoretic smoothing splines are approximate linear filters, submitted 